\title{
Correction to: Cognitive Behavioral Approach to Stress
}

\author{
Correction to: \\ Chapter 7 in: K. B. Koh, Stress and Somatic Symptoms, \\ https://doi.org/10.1007/978-3-030-02783-4_7
}

The published version of this book had missed to include the reference number [11] as follows: 7.6.2 Examples of Interventions [11] in page 78. This has now been updated. 